\title{
Belgeo
}

Revue belge de géographie

$4 \mid 2001$

Miscellaneous

\section{Multimodalité TGV-avion : considérations sur le cas de Bruxelles}

Multimodality TGV-airplane: some considerations on the Brussels case

\section{Frédéric Dobruszkes}

\section{(2) OpenEdition}

1 Journals

Édition électronique

URL : https://journals.openedition.org/belgeo/15666

DOI : 10.4000/belgeo.15666

ISSN : 2294-9135

Éditeur :

National Committee of Geography of Belgium, Société Royale Belge de Géographie

\section{Édition imprimée}

Date de publication : 30 décembre 2001

Pagination : 335-350

ISSN : 1377-2368

\section{Référence électronique}

Frédéric Dobruszkes, « Multimodalité TGV-avion : considérations sur le cas de Bruxelles », Belgeo [En ligne], 4 | 2001, mis en ligne le 31 décembre 2001, consulté le 15 novembre 2021. URL : http:// journals.openedition.org/belgeo/15666; DOI : https://doi.org/10.4000/belgeo.15666

Ce document a été généré automatiquement le 15 novembre 2021.

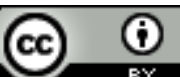

Belgeo est mis à disposition selon les termes de la licence Creative Commons Attribution 4.0 International. 


\section{Multimodalité TGV-avion : considérations sur le cas de Bruxelles}

Multimodality TGV-airplane: some considerations on the Brussels case

Frédéric Dobruszkes

\section{Introduction : TGV et desserte des aéroports}

\section{Exemples d'interconnexion et d'intégration des réseaux}

Paris CDG

1 Le cas de l'aéroport Paris Charles de Gaulle (CDG) est l'illustration parfaite de l'interconnexion des réseaux $\mathrm{TGV}^{1}$ et aériens, allant plus loin qu'une simple juxtaposition de réseaux puisque certains vols courts courriers sont remplacés par des TGV dans le cadre d'une offre et d'une tarification intégrées. C'est ainsi qu'outre de nombreux TGV français, 5 aller-retour Thalys Bruxelles-Midi-Paris CDG (sous numéro de vol Air France mais accessibles à tous) alimentent quotidiennement cet aéroport, où se situe le principal hub d'Air France, en lieu et place d'avions Air France. Les clients Air France transitant sur ces trains échangent leur billet d'avion Paris - Bruxelles ou Bruxelles-Paris contre un billet d'embarquement Thalys (première classe). Des accords ont également été signés entre Air France et la SNCF concernant certains trajets intrafrançais en TGV.

2 Un hub (de l'anglais « moyeu ») est un point central dans un réseau " hub and spokes », modèle de concentration de l'offre d'une compagnie ou groupe de compagnies aériennes sur un nombre réduit d'aéroports. Le(s) hub(s) y sont organisés en platesformes de correspondance entre vols. Les vols peu ou pas rentables sont supprimés, le réseau prend une forme uni ou pluri-étoilée (fig. 1) et les voyageurs, selon les points de 
vue, soit subissent des correspondances, soit au contraire disposent de correspondances mieux organisées (« opportunités de correspondance »).

Figure 1. Structure morphologique d'un réseau avec hub.

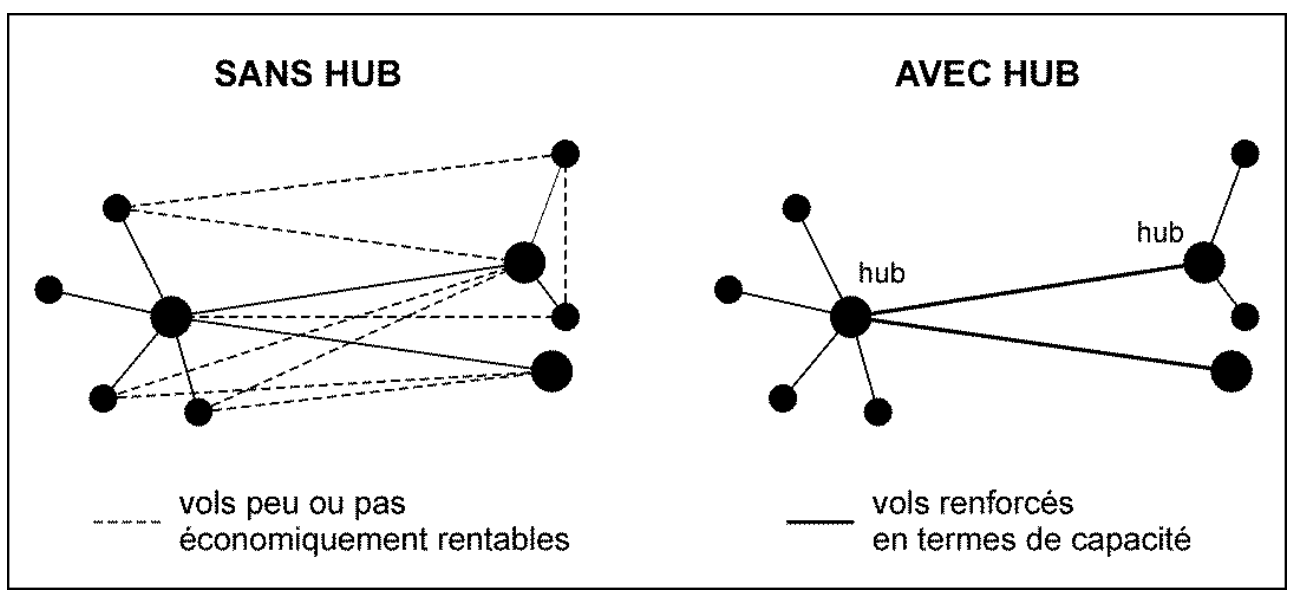

Du point de vue d'Air France, les Thalys desservant Paris CDG jouent clairement un rôle de vol court courrier alimentant son hub - l'un des plus importants en Europe voire dans le monde - en visant une correspondance appropriée avec les vols longs courriers.

D'un point de vue économique et logistique, Air France et Thalys International ont signé un accord en vertu duquel la première réserve (et paie) toutes les places de un ou deux wagons selon les trains et les jours, quel que soit le taux de remplissage effectif et avec possibilité de réservation supplémentaire. Le risque est ainsi partagé entre les deux sociétés, tandis qu'Air France réalise une intéressante opération financière car, pour la distance considérée, le coût de mise en ligne d'un TGV est inférieur à celui d'un avion. De plus, Air France peut tenter d'attirer une clientèle nouvelle qui en avion ou par route n'aurait pas opté pour Paris CDG, d'autant plus que pour le client le prix du billet combiné TGV + avion est moins élevé que le prix des billets acquis séparément. D'un point de vue coût pour la compagnie, le seul élément connu est que le déficit de la ligne s'en est trouvé réduit.

5 Outre Air France, United Airlines, American Airlines et Lufthansa ont également signé avec la SNCF des accords concernant Paris CDG («TGV Air »). Notons que dans tous les cas c'est à l'opérateur aérien que le voyageur achète son billet combiné.

\section{Francfort}

6 À Francfort, la desserte à grande vitesse de l'aéroport est de mise depuis l'inauguration en 1999 du nouvel " AIRail Terminal », desservi à cadence horaire par quatre relations avant tout nationales, dont trois à grande vitesse (ICE) ${ }^{2}$. Comme à Paris CDG, la gare est très bien implantée par rapport au terminal aérien, et les correspondances se font d'autant plus aisément qu'un check-in a spécialement été aménagé au plus près de celle-ci pour les voyageurs arrivant en train à l'aéroport ${ }^{3}$.

7 La desserte de l'aéroport par les trains à grande vitesse est clairement perçue comme une opportunité d'accroître son aire d'influence dans un contexte de concurrence entre les grands hubs européens tout en améliorant la capacité d'accueil de l'aéroport puisque parkings, accès routiers voire bureaux de check-in/out peuvent être délocalisés dans les gares à travers le pays ${ }^{4}$. Avec la libération de certains créneaux 
horaires aériens (vols courts courriers), cette intermodalité doit donc permettre à l'aéroport de Francfort d'assurer sa croissance.

Dans un tel contexte et sachant que d'ici une décennie une grande partie du territoire allemand (ainsi que néerlandais et belge) sera à moins de trois heures en ICE de cet aéroport, on ne s'étonnera pas de découvrir le slogan «by train from all region of Germany and by plane to any destination around the world».

Depuis mars 2001, une expérience pilote est menée par Lufthansa et la Deutsche Bahn entre la gare centrale de Stuttgart et l'aéroport de Francfort («AIRail service»). Sont proposés aux voyageurs inter-modaux: voyage première classe en train à grande vitesse ( 7 services par jour et par sens), intégration tarifaire, horaires coordonnés, informations intégrées tout au long de la chaîne de déplacement et, surtout, check-in et check-out en gare de Stuttgart, en ce compris pour les bagages. Les voyageurs n'ont donc pas à porter leurs bagages lors des accès aux quais et dans le train.

\section{Lyon St-Exupéry}

Depuis 1994, cet aéroport est desservi par des TGV contournant Lyon et desservant principalement Paris, le nord, l'ouest et le sud de la France. C'est le seul aéroport français autre que Paris directement desservi par TGV. Toutefois, l'offre y a été réduite à l'occasion de la mise en service du TGV Méditerranéenne au sud de Valence (juin 2001), les TGV concernés passant dorénavant par Lyon ville. On ne dénombre ainsi à l'aéroport de Lyon que 14 TGV par jour, exclusivement de/vers Paris et Grenoble ou Chambéry, contre 58 à Paris CDG, de/vers la Belgique et le nord, l'ouest, le sud et le sudest de la France ${ }^{5}$.

11 Aucun accord n'a été signé entre la SNCF et les compagnies aériennes concernant le pré- ou post-acheminement TGV des voyageurs vers et depuis Lyon St-Exupéry.

\section{Des résultats contrastés}

\section{Un certain succès à Paris CDG}

12 En 1999, soit avant la mise en service des Thalys précités, la gare TGV de Paris CDG était fréquentée par 1,3 millions de voyageurs, dont $2 / 3$ en correspondance TGV/avion, soit $3 \%$ des usagers land side de cet aéroport (Air France, 2000).

13 L'offre Thalys + Air France a attiré environ 70000 voyageurs en 2001 et l'offre " TGV Air » entre 40000 et 50000 voyageurs annuels en 2000 et 2001.

14 Les potentialités semblent loin d'être épuisées si l'on sait que l'on comptait, en 2000, près de 729000 voyageurs aériens embarquant à Lyon pour Paris CDG ou Orly ${ }^{6}$.

\section{Le succès rapide à Francfort}

En termes d'impact sur la demande, la mise en œuvre de l'AIRail terminal a eu les conséquences suivantes (IARO, 2001) ${ }^{7}$ :

- part des trains grandes lignes dans le marché de l'accès land side à l'aéroport passant de $7 \%$ en 1998 à $15 \%$ en 2000 (soit sensiblement plus qu'à Paris CDG), le volume de voyageurs transportés par ceux-ci ayant doublé ;

- léger accroissement de l'aire de recrutement de la clientèle de l'aéroport, en particulier audelà de $100 \mathrm{~km}$. 


\section{et appelée à un avenir certain d'autant que les chiffres avancés ici ne correspondent} qu'à une période courte par rapport à l'inauguration de l'AIRail terminal.

Il est par ailleurs trop tôt pour disposer de résultats concernant les améliorations mises en œuvre en 2001 sur la liaison avec Stuttgart. On sait cependant que l'expérience devrait être étendue en 2002 sur les relations vers ou de Cologne et Düsseldorf.

\section{L'échec de Lyon}

En 1997, seuls $10 \%$ des 110000 usagers de la gare TGV étaient en correspondance TGV/ avion, soit $0,2 \%$ des voyageurs land side. Les autres voyageurs étaient pour une grande partie des habitants des départements de l'est de Lyon qui trouvaient plus pratique de se rendre en voiture à la gare de l'aéroport qu'à celle de la ville (IAURIF, 1999).

19 En 2001, la fréquentation annuelle de la gare est estimée à 237000 voyageurs ${ }^{8}$. La part des voyageurs en correspondance TGV/avion n'a pu être connue, témoignant peut être d'une gêne dans le chef des autorités responsable quant à une interconnexion attirant peu de clientèle.

20 Ce contraste avec Paris CDG et Francfort se comprend bien dans un contexte où ces derniers sont des aéroports d'envergure nationale et internationale, disposant dès lors d'une aire d'influence compatible avec les distances TGV. À l'inverse, Lyon est un aéroport de province avec une offre limitée, tandis que la ligne TGV est orientée nordsud, donc vers des régions dotées d'aéroports concurrents (Marseille et surtout Paris).

21 Notons que dans la mesure où une ligne à grande vitesse contournant Lyon était créée, y prévoir une gare à l'aéroport demeure une solution réservant l'avenir. À ce propos, la récente décision en faveur d'un troisième aéroport « parisien », à $125 \mathrm{~km}$ au nord de la capitale, peut s'interpréter de manière contrastée. D'une part, Lyon ne sera pas ce troisième aéroport, comme d'aucuns l'avaient imaginé. Mais d'autre part, une partie de l'offre aérienne dite parisienne sera orientée sur ce nouvel aéroport, plus éloigné du sud que ne l'est Paris CDG. Lyon St-Exupéry y regagnerait-il alors des voyageurs?

\section{Pourquoi desservir un aéroport par TGV?}

L'échec du cas de Lyon Saint-Exupéry laisse penser qu'une certaine prudence s'impose en matière d'interconnexion TGV/aéroports. Ceci nous amène à poser la question des motivations qui peuvent inciter à desservir un aéroport par des TGV. Nous en avons identifié quatre :

1. faciliter l'accès à l'aéroport concerné, pour des voyageurs suffisamment éloignés pour justifier le recours au TGV ;

2. transférer sur le rail les voyageurs de vols courts courriers qui alimentent les vols moyens ou longs courriers ${ }^{9}$, vols courts courriers qui tendent à «encombrer" les compagnies (économiquement) et les aéroports (occupation de slots, dans un contexte de capacité limitée);

3. augmenter l'attractivité d'un aéroport par rapport à des aéroports concurrents, afin d'y attirer la clientèle localisée dans des aires d'influence se superposant ;

4. implanter une gare TGV «bis », en banlieue suffisamment dense en activités et/ou habitat, éventuellement sur un itinéraire de contournement d'agglomération (par exemple Massy dans la banlieue sud de Paris); compte tenu du nombre limité de gares que l'on peut 
implanter sur une ligne TGV, coupler gare d'aéroport et gare de banlieue apparaît comme une solution rationnelle.

23 On peut se demander si le TGV est alors complémentaire ou concurrent de l'offre aérienne. La réponse dépend des modalités d'organisation des offres respectives et des acteurs considérés :

- du point d'une compagnie aérienne donnée, si l'offre TGV est organisée de manière concertée avec son offre aérienne et que l'offre train + avion est vendue comme un produit unique, il faut y voir une complémentarité TGV/avions ;

- une compagnie concurrente implantée dans l'aéroport desservi par TGV peut également bénéficier d'un apport supplémentaire de voyageurs et, même en l'absence de produit unique train + avion, le juger comme un complément à son offre aérienne à condition qu'il ne diminue pas le taux de remplissage de ses avions courts courriers volant sur des relations TGV;

- du point de vue d'une compagnie aérienne implantée dans un aéroport non-desservi par TGV, ce dernier sera vu comme un concurrent si des TGV partent de la ville voisine pour d'autres grands aéroports (ex. : l'offre Air France \& Thalys Bruxelles-Midi - Paris CDG - Dakar était concurrente de l'offre Sabena Bruxelles-National - Dakar, le prix du billet étant équivalant voire légèrement inférieur via Paris).

Qu'en est-il dans le cas de Bruxelles-National ? Cet aéroport requiert-il une desserte TGV ? Nous nous proposons d'apporter des éléments de réponse, compte tenu de l'offre et la demande actuelles, des évolutions plausibles et des enjeux politiques et environnementaux.

\section{Le cas de Bruxelles-National}

\section{Analyse de l'offre et de la demande actuelles}

\section{Aspects méthodologiques}

\section{offre}

Poser la question d'une desserte TGV de Bruxelles-National conduit tout d'abord à s'interroger sur l'origine ou la destination géographique des voyageurs qui transiteraient par le réseau TGV. La littérature et différentes études s'accordent pour fixer à 2,5 heures le temps de parcours au-delà duquel les parts de marché du train décroissent fortement face à l'avion. Par ailleurs, le TGV n'a guère de sens qu'au-delà d'une heure de trajet.

En supposant une vitesse commerciale de $200 \mathrm{~km} / \mathrm{h}^{10}$, ceci établit la zone d'utilité potentielle d'une desserte TGV de Bruxelles-National à des distances variant de 200 à $500 \mathrm{~km}$.

Dans un tel rayon d'action et en tenant du compte du réseau TGV projeté à l'horizon 2010, il apparaît que Bruxelles-National est concurrencé par des aéroports dont l'offre, en termes de destinations mais aussi de fréquences, est nettement plus développée qu'à Bruxelles-National.

Nous commencerons dès lors par comparer l'offre de Bruxelles-National à celle des aéroports situés à des temps de parcours variant de 1 à 2,5 heures en TGV. Ceci afin de cerner les supériorités éventuelles de l'offre de Bruxelles-National, qui seraient autant 
de raisons « objectives » à ce que des voyageurs aériens y arrivent de loin en TGV pour des correspondances TGV/avion.

En excluant les petits aéroports régionaux dont l'offre est actuellement faible ${ }^{11}$, les aéroports qui nous intéressent sont ceux repris au tableau 1.

Tableau 1. Aéroports comparés.

\begin{tabular}{ll} 
Code IATA & Aéroport \\
\hline BRU & \\
CDG & Bruxelles-National (Zaventem) \\
ORY & Paris Charles de Gaulle (Roissy) \\
LIL & Paris Orly \\
AMS & Lille \\
CGN & Amsterdam (Schiphol) \\
DUS & Köln/Bonn \\
FRA & Düsseldorf \\
LGW & Francfort \\
LHR & Londres Gatwick \\
STN & Londres Heathrow
\end{tabular}

La variable de comparaison est le nombre de jours par semaine où une desserte est offerte, par vols réguliers ${ }^{12}$ et sans correspondances. Deux séries de comparaisons ont été menées, sur base des horaires de l'hiver 2000-2001:

- analyse des exclusivités de Bruxelles-National indépendamment des fréquences offertes (tableau 3);

- analyse du différentiel de fréquences offertes, à l'échelle de la semaine et uniquement pour les destinations communes à Bruxelles-National et à l'aéroport comparé (par exemple, Bruxelles offrait des relations vers Kinshasa 6 jours sur 7 , contre 1 jour sur 7 au départ de Paris - des voyageurs français pourraient donc être tentés d'embarquer à BruxellesNational) (tableau 4).

31 Les aéroports allemands de l'ouest, parisiens et londoniens ont été respectivement regroupés en trois aéroports fictifs et les fréquences recalculées en conséquence.

\section{Demande}

Ensuite, une analyse de la demande a été tentée, dans la mesure des données disponibles, afin de vérifier si des voyageurs aériens viennent effectivement de l'étranger pour embarquer à Bruxelles-National.

\section{Analyse de l'offre}

\section{Nombre de destinations}

Le nombre de destinations régulières offertes par les aéroports ou groupes d'aéroports retenus figure au tableau 2. 
Tableau 2. Nombre de destinations régulières (hiver 2000-2001).

Origine Nombre

$\begin{array}{lc}\text { Bruxelles-National } & 147 \\ \text { Allemagne (Francfort + Cologne + Düsseldorf) } & 237 \\ \text { Amsterdam (Schiphol) } & 210 \\ \text { Lille } & 15 \\ \text { London (Heathrow + Gatwick + Stansted) } & 271 \\ \text { Paris (Charles de Gaulle + Orly) } & 237\end{array}$

Une destination disposant de plusieurs aéroports n'est comptée qu'une seule fois (ex. : Milano Linate et Milano Malpensa)

Source : analyse sur base des annuaires OAG

\section{Exclusivités de Bruxelles-National}

Le tableau 3 compare les exclusivités qu'offre Bruxelles-National par rapport aux aéroports comparés, en distinguant les continents de destination. On constate que celles-ci sont limitées en nombre (Lille exceptée, à conditions de ne pas tenir compte de Paris CDG), et concernent surtout des destinations d'Europe et d'Afrique (en fait l'Afrique Noire).

Tableau 3. Nombre d'exclusivités au départ de Bruxelles-National, par continent (hiver 2000-2001).

\begin{tabular}{lccccccc} 
Par rapport à & Afr. & Am-N & Am-L & Asie & Eur. & M-Ori. & Total \\
\hline Allemagne & & & & & & & \\
(Francfort + Cologne + Düsseldorf) & 23 & 2 & 1 & 0 & 21 & 4 & 51 \\
Amsterdam (Schiphol) & 20 & 1 & 0 & 2 & 13 & 3 & 39 \\
Lille & 30 & 11 & 1 & 7 & 79 & 9 & 137 \\
Lille + Paris Charles de Gaulle * & 10 & 2 & 1 & 2 & 14 & 3 & 32 \\
London & 19 & 2 & 1 & 0 & 13 & 3 & 38 \\
(Heathrow + Gatwick + Stansted) & 7 & 2 & 1 & 2 & 11 & 3 & 26
\end{tabular}

Seuls les vols réguliers sont pris en compte. Une destination disposant de plusieurs aéroports n'est comptée qu'une seule fois (ex. : Milano Linate et Milano Malpensa).

${ }^{*}$ Compte tenu de la proximité Paris CDG-Lille en TGV.

Source : analyse sur base des annuaires OAG

Il faut par ailleurs préciser que certaines exclusivités de Bruxelles-National sont des exclusivités uniquement liées à la distance (Bruxelles-National offre par exemple des relations vers Birmingham qui n'existent pas au départ de Londres).

\section{Fréquences supérieures à Bruxelles-National}

Comme pour les destinations exclusives, la supériorité de Bruxelles-National en termes de fréquences hebdomadaires, par rapport aux aéroports considérés, concerne presque exclusivement des destinations européennes et africaines (y compris l'Afrique du nord cette fois) (tableau 4). Ces destinations sont toutefois fortement limitées en nombre. 
Tableau 4. Nombre de destinations plus fréquentes au départ de Bruxelles-National (à l'échelle de la semaine, par continents, uniquement pour les destinations communes) (hiver 2000-2001).

\begin{tabular}{|c|c|c|c|c|c|c|c|}
\hline Par rapport à & Afr. & $A m-N$ & $A m-L$ & Asie & Eur. & M-Ori. & Total \\
\hline \multicolumn{8}{|l|}{ Allemagne } \\
\hline (Francfort + Cologne + Düsseldorf) & 3 & & & 1 & 2 & & 6 \\
\hline Amsterdam (Schiphol) & 4 & & & & 6 & & 10 \\
\hline Lille & & & & & 1 & & 1 \\
\hline $\begin{array}{l}\text { Lille + Paris Charles de Gaulle* } \\
\text { London }\end{array}$ & 3 & & & & 3 & & 6 \\
\hline (Heathrow + Gatwick + Stansted) & 3 & & & & 3 & & 6 \\
\hline Paris (Charles de Gaulle + Orly) & 2 & & & & 3 & & 5 \\
\hline
\end{tabular}

Seuls les vols réguliers sont pris en compte. Une destination disposant de plusieurs aéroports n'est comptée qu'une seule fois (ex. : Milano Linate et Milano Malpensa).

*Compte tenu de la proximité Paris CDG - Lille en TGV.

Source : analyse sur base des annuaires OAG

\section{Analyse de la demande}

Si Bruxelles-National offre donc un certain nombre de destinations en exclusivité ou des fréquences hebdomadaires plus attractives, encore faut-il vérifier si cela intéresse des voyageurs venant de/allant vers la zone de desserte TGV retenue.

\section{Voyageurs land side en provenance de l'étranger}

D'après une enquête menée par un bureau d'études auprès des voyageurs land side ${ }^{13}$ les 6 et 10 juin 1997, 5,1 \% d'entre eux venaient de l'étranger, sans précision géographique autre que le pays concerné (RVA/Tritel, 1998).

Toutefois, méthodologiquement, on ne peut que s'interroger sur la représentativité d'une enquête menée seulement durant deux journées d'une même semaine, alors que par essence les origines et destinations varient fortement tant à l'échelle de l'année que de la semaine.

\section{Voyageurs en transfert air/air à Bruxelles-National}

Nous avons reçu de BIAC - gestionnaire de l'aéroport - les statistiques des passagers en transfert ${ }^{14}$ air/air à Bruxelles-National en 2000, selon leur destination. L'origine n'est pas connue et il nous est donc malheureusement impossible de croiser origine et destination pour établir une matrice exhaustive. Plusieurs enseignements intéressants peuvent néanmoins être tirés de ces données qui nous donnent une indication de l'organisation actuelle de la demande exprimée.

41 Par destination, les voyageurs en transfert à Bruxelles-National représentaient (en 2000) 3387700 unités. Pour être comparés aux statistiques annuelles de fréquentation de l'aéroport [ = départs + arrivées], il faut multiplier ce chiffre par 2, ce qui nous donne $31 \%$ de voyageurs en transfert (ce taux était de $19 \%$ en 1996 et cette évolution traduit vraisemblablement l'augmentation de la fonction hub de Bruxelles-National, jusqu'à la récente faillite de la Sabena).

La fig. 2 représente la destination des voyageurs en transfert. On peut y constater :

- l'importance écrasante des destinations européennes, surtout occidentales: $81 \%$ des transferts ; 
- le poids de plusieurs grandes villes relativement proches et intégrées au réseau TGV : Paris (107 000), Londres (100 500) et Amsterdam (85 100);

- le faible poids des destinations exclusives par rapport aux aéroports comparés, et des destinations lointaines en général (par exemple : 266500 voyageurs à destination de toute l'Afrique Noire soit $8 \%$ des transferts), New York excepté.

Figure 2. Destination des passagers en transfert air/air à Bruxelles-National (2000).

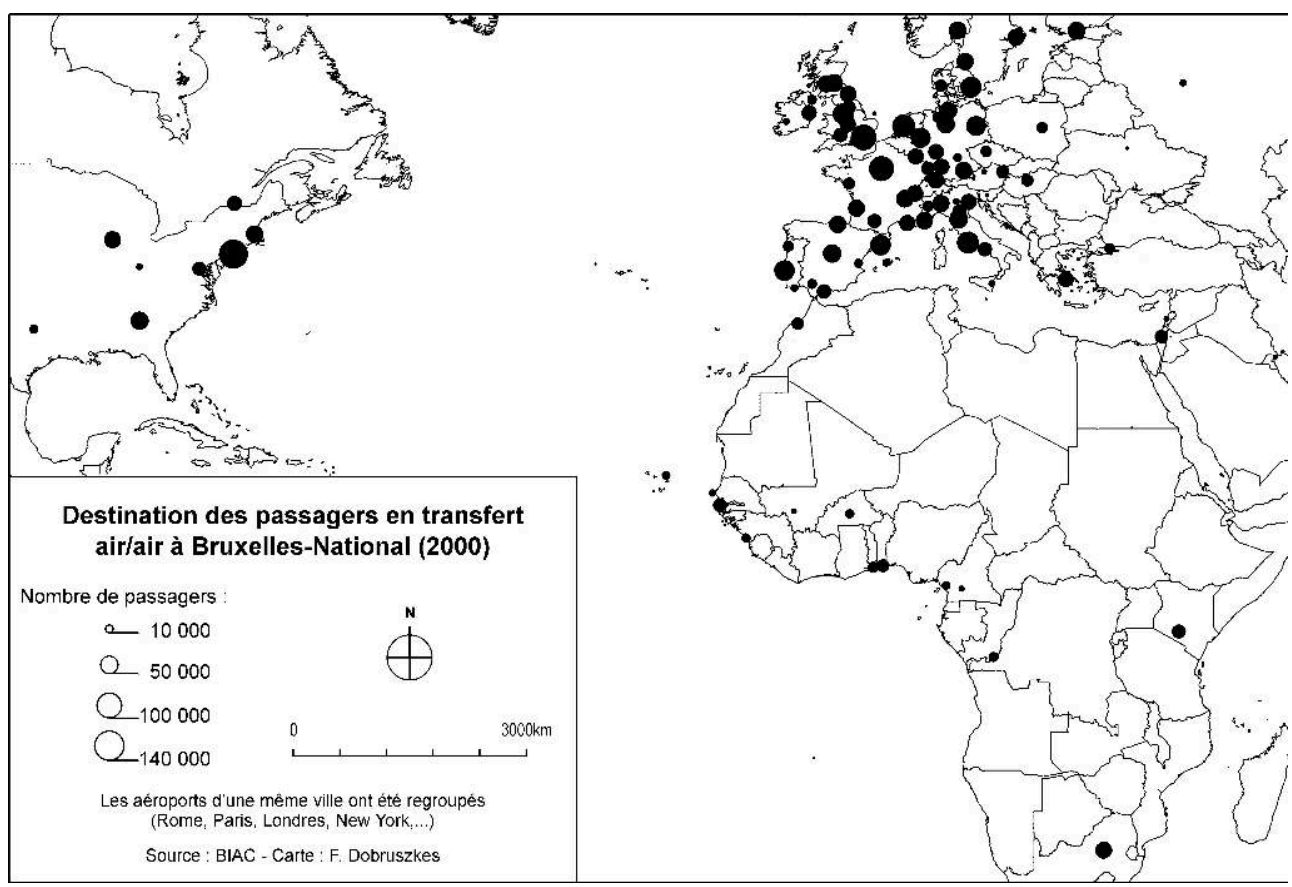

Cette carte relativise l'idée souvent répandue selon laquelle les transferts ont avant tout lieu entre courts courriers et longs courriers. Si tel était le cas, la surface totale des cercles lointains devrait être semblable à celle des cercles plus proches.

On peut en déduire que la demande de transport avion + TGV à Bruxelles-National est actuellement faible puisque :

- c'est en liaison avec les vols longs courriers que les rabattements par TGV sont les plus intéressants : on imagine assez mal un Néerlandais venir en TGV à Bruxelles-National pour y prendre un avion le menant en Suisse. Une enquête menée en gare TGV de Paris CDG confirme que l'intermodalité TGV/avion y a d'autant plus de succès que les destinations aériennes sont lointaines (IAURIF, 1999).

- le poids des destinations compatibles avec le rayon d'action des TGV semble faible. Ainsi, les 107000 voyageurs à destination de Paris équivalent, en supposant une équi-répartition dans le temps à l'échelle de l'année, à moins de 300 voyageurs par jour, à répartir dans plusieurs trains. Avec une hypothèse de 8 trains par jour (soit un train toutes les deux heures), une équi-répartition ne donnerait que 37 voyageurs par train. Si l'on se limite à 5 trains par jour, correspondant aux 5 principales vagues d'atterrissages et de décollages avant faillite de la Sabena (fig. 3), ont atteint 59 voyageurs par train, ce qui reste peu. 
Figure 3. Nombre d'arrivées/départs de vols réguliers le 25/7/2001 à Bruxelles-National selon I'horaire officiel.

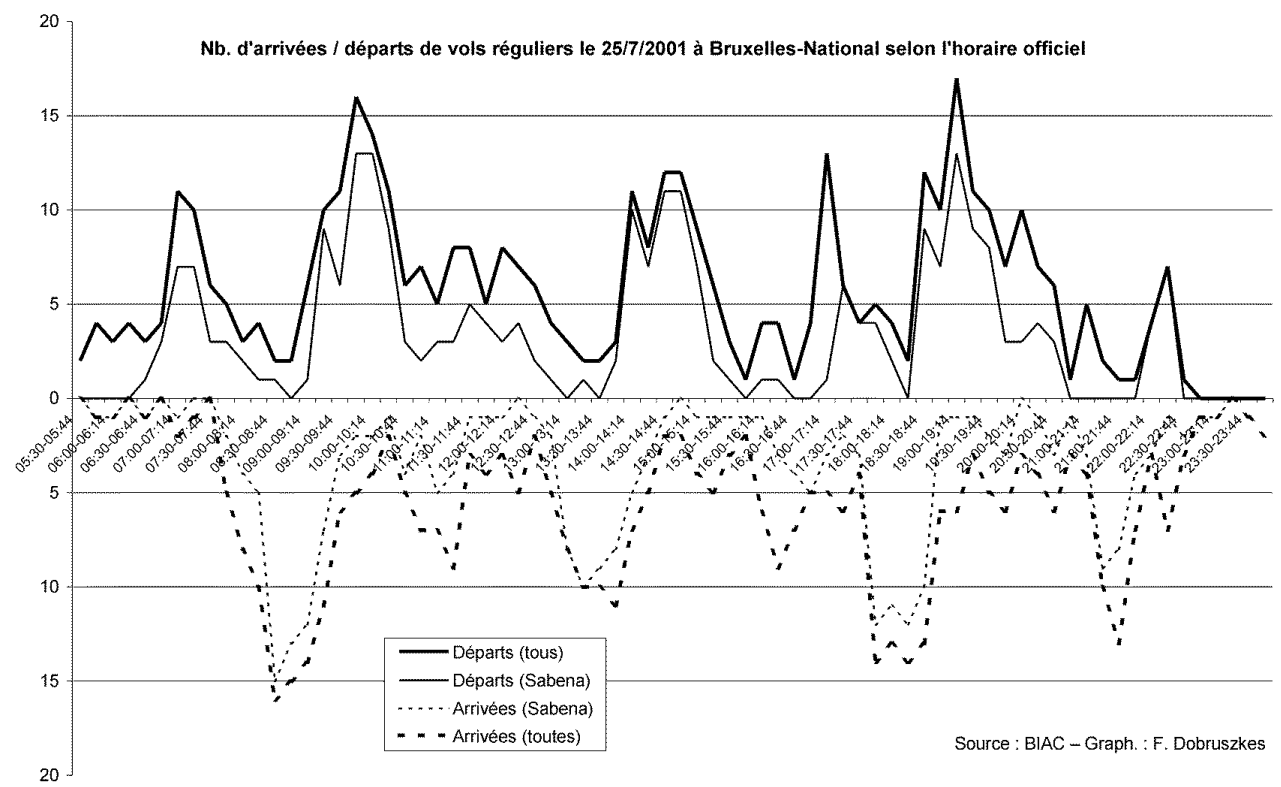

\section{Conclusions}

On peut déduire des éléments précédents que du seul point de vue de l'offre et de la demande actuelles, une interconnexion TGV/avion à Bruxelles-National n'est sans doute pas requise à court voire moyen terme.

Ceci est toutefois à valider en analysant plus sérieusement l'origine géographique des voyageurs land side d'une part, et la répartition dans le temps des voyageurs en transferts air/air afin d'en déduire les taux de remplissage de TGV qui desserviraient Bruxelles-National.

Il faut par ailleurs rappeler que les données d'offre analysées correspondent à l'hiver 2000-2001, soit avant la faillite de la Sabena en novembre 2001 et son remplacement par une compagnie (DAT) plus modeste en termes de dessertes proposées. Au moment de rédiger cet article, nous nous trouvons dans une situation intermédiaire entre une offre passée et une offre future dont on ne connaît pas les caractéristiques, celles-ci étant notamment fonction des compagnies aériennes qui prendront, ou ne prendrons pas, la place laissée libre par la Sabena et la DAT si celle-ci ne prend pas une envergure intercontinentale.

\section{Aspects politiques et environnementaux}

Si l'offre et la demande avant la faillite de la Sabena ne justifiaient sans doute pas une desserte TGV de Bruxelles-National, la situation pourrait changer, en fonction de plusieurs évolutions possibles :

- l'augmentation générale de la demande de transport ;

- l'expression d'une demande nouvelle qui serait suscitée par une desserte TGV de l'aéroport, du fait des nouvelles conditions de l'offre en termes de temps de parcours et de tarifs ;

- la concurrence croissante entre aéroports, pour attirer les voyageurs situés entre deux (par exemple des Lillois hésitant entre Bruxelles-National et Paris CDG). 
Ceci pose deux questions qui sont des choix de société à opérer et sont donc d'ordre politique.

1. Faut-il permettre à la demande latente de s'exprimer et à la demande en général d'augmenter? Autrement dit, jusqu'à quel stade faut-il accepter que les gens se déplacent compte tenu des impacts environnementaux suscités ${ }^{15}$ ? Cette question va bien entendu à contresens du discours dominant, qui appelle à répondre positivement à l'augmentation de la demande, dont on ne sait en fait pas trop dans quelle mesure elle n'est pas elle-même suscitée par l'augmentation de l'offre justifiée par l'augmentation prédite de la demande...

- Si les pouvoirs publics se dégagent assez massivement de la fonction de transporteur aérien, il leur reste en tout cas, s'ils le veulent bien, la possibilité de définir le niveau d'offre acceptable pour la collectivité, notamment au travers des infrastructures qu'ils gèrent et/ou qu'ils autorisent via les permis d'urbanisme et d'environnement délivrés.

2. Faut-il encourager la concurrence entre aéroports et, dans ce cadre, favoriser BruxellesNational? Autrement dit, faut-il chercher à faire transiter par Bruxelles-National des voyageurs sans rapport avec la région étendue de Bruxelles ou la Belgique, qui ne font que passer sans susciter d'autres valeurs ajoutées (financière, sociale, culturelle,...) que l'usage de l'aéroport, au bénéfice de son gestionnaire (privatisable) et d'un certain nombre d'emplois, mais sans influence notoire sur le rayonnement de la région ou du pays, sauf éventuellement indirectement par le nombre de destinations aériennes offertes?

- Si la réponse politique à cette question est oui, les régions intéressantes sont probablement celles de Lille, du sud des Pays-Bas et d'Aix la Chapelle. Il faut toutefois être conscient que des TGV desservent ou desserviront aussi les aéroports de Paris CDG, Schiphol, Francfort, Cologne et Düsseldorf. Citons aussi pour mémoire l'éventualité1 ${ }^{16}$, à plus long terme, d'un troisième aéroport «parisien » entre Paris et Lille, près de Chaulnes, desservi par le TGV Nord.

S'ajoutent à cela deux questions complémentaires :

1. Faut-il favoriser l'implantation d'activités économiques à proximité immédiate des aéroports, au point d'en faire des «aérovilles» (Varlet, 1992 et 1997), suivant en cela certaines tendances (cf. Amsterdam, Genève, Francfort, Londres, Zürich, Paris CDG,...), ou vaut-il mieux ne privilégier des développements que dans la ville centre?

- Si le choix de l'aéroville est opéré à Bruxelles-National, alors desservir au mieux un tel pôle conduira sans doute à favoriser son développement, encore qu'en la matière les effets sont loin d'être automatiques (Offner, 1993), tout en incitant les hommes d'affaires à opter pour le TGV plutôt que l'avion. Inversement, une aéroville suffisamment importante est à même de justifier une gare TGV de banlieue pour son trafic propre, gare qui profiterait alors également à l'aéroport et à la banlieue nord-est de Bruxelles dans son ensemble.

2. Faut-il favoriser la DAT succédant à la Sabena, compagnie nationale historique, malgré le contexte de dérégulation et de privatisation qui caractérise le secteur aérien?

- En cas de réponse positive, des dessertes TGV de l'aéroport bruxellois pourraient aider la compagnie belge à remplir ses avions, sous réserve d'une demande suffisante ce qui reste à prouver comme nous l'avons vu plus haut.

\section{Contraintes à prendre en compte}

\section{Contraintes d'infrastructures ferroviaires}

51 À court et moyen termes, les infrastructures ferroviaires sont insuffisantes, aux heures de pointe, pour absorber un trafic supplémentaire entre Bruxelles et l'aéroport. La ligne 36 sera portée à 4 voies entre Bruxelles et Louvain d'ici 2008, permettant alors 
une meilleure séparation des trains lents et rapides et une augmentation du nombre de trains y circulant.

Se pose par ailleurs le problème de la gare en cul-de-sac à l'aéroport. Pour que des TGV Belgique-Allemagne ou Pays-Bas puissent passer par celui-ci, l'infrastructure « diabolo » doit être réalisée, afin de le connecter directement aux lignes d'Anvers et Liège (fig. 4). Il est à noter que le « diabolo » se justifiant par les seuls besoins du seul réseau intérieur, le passage de TGV à Bruxelles-National n'implique donc pas de travaux d'infrastructure non-requis par ailleurs.

Figure 4. Infrastructures ferroviaires requises.

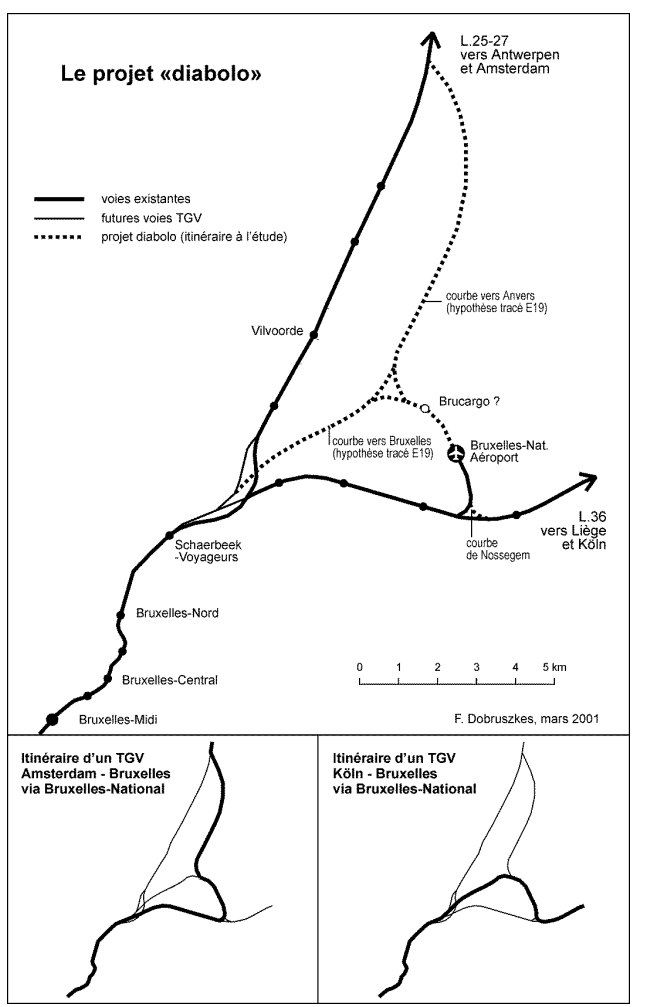

\section{Contraintes d'exploitation et contraintes économiques}

Si le projet « diabolo » permet effectivement le détournement de trains via BruxellesNational, il faut être conscient qu'il engendrerait une perte de temps de parcours significative pour les trains concernés et donc pour les voyageurs non-intéressés par l'aéroport ${ }^{17}$.

On peut estimer que les TGV qui seraient déviés via Bruxelles-National perdront environ 15 à 20 minutes sur le trajet Köln-Bruxelles ou Amsterdam-Bruxelles, compte tenu de l'allongement de distance, des courbes dans le tracé et de l'arrêt en gare. Ce temps est à mettre en balance avec les réductions de temps qui seront rendues possibles grâce aux futures lignes à grande vitesse, respectivement 53 et 60 minutes.

Outre la perte de temps pour les usagers non concernés par Bruxelles-National, de moins bons temps de parcours auraient des répercutions financières négatives pour la $\mathrm{SNCB}$, car de ces temps dépendent la répartition des coûts et des recettes entre compagnies ferroviaires, conformément aux accords signés entre elles. D'un point de 
vue strictement financier, il s'agirait donc de compenser cette évolution par une vente conséquence de titres de transports, une intervention financière des pouvoirs publics étant légalement impossible dans le cadre actuel ${ }^{18}$.

Par ailleurs, la dégradation des temps de parcours (s'agissant de TGV Allemagne/PaysBas - Belgique) ou l'augmentation de ceux-ci (pour les TGV France-Bruxelles qui seraient prolongés jusqu'à Bruxelles-National) implique vraisemblablement l'acquisition de matériel roulant supplémentaire, dont le coût ne peut être négligé ${ }^{19}$.

\section{Contraintes de saturation aérienne}

L'évolution vers une situation de saturation de certains aéroports peut rendre tentant voire obligatoire le remplacement des vols courts courriers par des TGV. Si ceux-ci concernent des voyageurs se déplaçant entre deux villes suffisamment proches, le report sur des TGV inter-villes semble évident.

Par contre, si ces vols sont également utilisés par des voyageurs en transfert air/air, alors une desserte TGV de Bruxelles-National pourrait prendre plus de sens, dans un contexte où l'on prévoyait, avant la faillite de la Sabena, une saturation à l'horizon 2005-2010 en supposant un accroissement constant de la demande et l'absence d'investissement visant à augmenter la capacité de l'aéroport (COFAR - Centre Interuniversitaire d'Étude de la Mobilité, 2000). Toutefois, cette composante du débat revient à poser la question du caractère supportable de l'accroissement de la demande, puisque décharger les aéroports des vols courts courriers conduirait à libérer des créneaux horaires pour les vols moyens et longs courriers.

\section{Conclusions}

Il n'est pas facile de trancher la question de l'utilité d'une desserte TGV de BruxellesNational. Il faudrait en tout cas procéder à une enquête conséquente et suffisamment représentative de l'origine géographique des voyageurs land side, afin de compléter les données sur les transferts air/air.

Les arguments potentiellement favorables sont :

- un transfert modal au profit du chemin de fer, moins préjudiciable pour l'environnement que l'avion, à volume de demande inchangé ;

- si on l'estime nécessaire, le renforcement de Bruxelles-National comme hub comptant à l'échelle des aéroports européens et où les TGV feraient office de vols courts courriers;

- si on l'estime souhaitable, permettre à la demande latente de se réaliser et à la demande en général d'augmenter.

61 Les arguments défavorables sont :

- une clientèle apparemment limitée en volume, du moins dans l'état actuel des choses ;

- pour les trains de/vers les Pays-Bas et l'Allemagne, les pertes de temps significatives occasionnées aux voyageurs non-concernés par l'aéroport; ce seul aspect risque de limiter d'éventuels services TGV concernant Bruxelles-National à des relations de/vers la France ;

- les conséquences en termes de parc de matériel roulant, dont l'acquisition risque d'être jugée onéreuse eu égard au volume de clientèle. 
62

\section{BIBLIOGRAPHIE}

ACI, ATAG et IARO (2000), Airport rail links, guide to best practise, $114 \mathrm{p}$.

AIR FRANCE (2000), Rapport environnement 1999-2000, 48 p.

BIAC (2000), Bru trends 99, $44 \mathrm{p}$.

BIAC (2000), Rapport annuel 1999, 92 p.

BRUINSMA F., RIETVELD P. \& BRONS M. (2000), « Comparative study of hub airports in Europe: ticket prices, travel time and resheduling costs », Tijdschrift voor Economishe en Sociale Geografie, 91, 3, pp. 278-292.

COFAR - Centre Interuniversitaire d'Étude de la Mobilité (2000), COFAR phase II - Theme 4 : Airport Systems, main report (final), 84 p. + annexes.

COFAR - Resource Analysis, MVA Limited (septembre 2000), Landside Accessibility and Ground Transport, research phase II, theme 2, draft report, $100 \mathrm{p}$. 
DENNIS N. (décembre 1994), « Airline hub operations in Europe », Journal of Transport Geography, 2, 4, pp. 219-233.

IAURIF (1999), Le développement de la grande vitesse ferroviaire en Europe - Concurrence ou complémentarité avec le transport aérien ?, $26 \mathrm{p}$.

IARO (2001), Air Rail 2001 - conference documentation, Londres.

MENERAULT P. \& STRANSKY V. (1999), « La face cachée de l'intermodalité - essai de représentation appliquée au couple TGV/Air dans la desserte de Lille ", Les Cahiers Scientifiques du Transport, 35, pp. 29-53.

MINISTÈRE [belge] DES COMMUNICATIONS ET DE L'INFRASTRUCTURE (2002), TGV et accès rapides à Bruxelles et son aéroport - Enjeux belges et modèles étrangers, Actes du colloque, 2 volumes.

OFFNER J.-M. (1993), « Les "effets structurants" du transport : mythe politique, mystification scientifique », L'Espace Géographique, 3, pp. 233-242.

PAVAUX J. (1991), Les complémentarités train/avion en Europe, ITA, Paris, 162p.

RVA/Tritel (juin 1998), Accessibilité land-side à l'aéroport de Bruxelles-National, phase 1, Etude d'orientation, rapport de synthèse, inédit, 11 p. + cartes.

$\mathrm{SNCB}$, Contribution de la SNCB à la politique d'accroissement de la mobilité par le rail - projet de plan décennal d'investissement 2001/2010 - document complémentaire.

ULB-IGEAT (avril 2001), Éléments pour l'analyse de l'opportunité d'une desserte TGV de l'aéroport de Bruxelles-National, rapport d'étude pour la Ministre fédérale de la mobilité et des transports, inédit, $36 \mathrm{p}$.

ULB-IGEAT (septembre 2001), Analyse de la faisabilité à court terme de liaisons TGV entre Paris et l'aéroport de Bruxelles-National, rapport d'étude pour la Ministre fédérale de la mobilité et des transports, inédit, $12 \mathrm{p}$.

VARLET J. (1992), L'interconnexion des réseaux de transport en Europe, ITA, Paris, 197 p.

VARLET J. (1997), « Les grands aéroports internationaux français : enjeux et retombées territoriales », Annales de Géographie, 593-594, pp. 155-182.

\section{NOTES}

1. Le terme TGV sera utilisé ici comme terme générique décrivant l'ensemble des systèmes ferroviaires à grande vitesse (TGV français, ICE allemand, Thalys, EuroStar,...).

2. Hambourg - Bâle, Hambourg - Stuttgart, Dresde - Passau et Berlin - Nuremberg.

3. À Paris CDG par contre, le voyageur arrivant en train doit se rendre avec ses bagages jusqu'aux traditionnels guichets d'enregistrement, éventuellement à plusieurs centaines de mètres de la gare.

4. Encore faudrait-il vérifier si la gare de l'aéroport n'attire pas à elle des voyageurs exclusivement ferroviaires usant dès lors des accès et parkings de l'aéroport pour atteindre celleci en voiture, comme c'est largement le cas à Lyon-Saint-Exupéry (ex-Satolas).

5. En semaine, à l'automne 2001. Source: guides horaires SNCF. Un train compté dessert généralement deux régions opposées, l'aéroport se trouvant entre deux. Du point de vue des aéroports, les chiffres indiqués peuvent donc être multipliés par deux pour connaître le nombre d'origine et de destinations qui lui sont connectées.

6. Source : aéroport de Lyon, Rapport d'activités 2000. 
7. Chiffres cités par H. Fakiner, membre de la Commission pour les systèmes intermodaux de l'aéroport de Francfort, au colloque Air Rail 2001.

8. Par extrapolation de la fréquentation des 11 premiers mois de l'année.

9. Les vols courts courriers dont la seule fonction est de relier deux villes, sans correspondance avec d'autres vols, sont pour certains d'entre eux destinés à être remplacés par des TGV ville ville, et non ville - aéroport.

10. La vitesse commerciale tient compte du temps de montée en puissance, des distances de freinage, de la durée des arrêts intermédiaires et des vitesses réellement pratiquées. Elle ne doit pas être confondue avec la vitesse de croisière.

11. Dortmund (DTM), Maastricht (MST), Rotterdam (RTM), Hahn (HHN), London Luton (LTN), London City Airport (LCY), Charleroi (CRL), Liège-Bierset (LGG) et Beauvais (BVA).

12. Les charters sont donc exclus.

13. Voyageurs arrivant à l'aéroport autrement qu'en avion.

14. Le transfert ne doit pas être confondu avec le transit :

- transfert : les voyageurs changent d'avion ( = correspondance);

- transit : l'avion fait une escale mais les voyageurs restent à bord.

15. Même si, évidemment, à niveau égal de mobilité, il vaut mieux que les voyageurs utilisent des TGV que des avions, plus polluants par rapport au volume de voyageurs $\mathrm{x}$ kilomètres et posant des problèmes de bruit plus importants.

16. La décision de principe est prise, mais compte tenu de l'échéance retenue (2015) et des incertitudes politiques généralement de mise pour un projet d'envergure et planifié sur le long terme, on peut selon nous s'autoriser à parler d'éventualité.

17. Signalons ici que des liaisons Pays-Bas - Bruxelles-National - Allemagne, évitant Bruxelles, n'ont sans doute pas de sens dans la mesure où la demande serait d'autant plus limitée qu'une relation TGV Amsterdam - Düsseldorf à $200-220 \mathrm{~km} / \mathrm{h}$ est prévue.

18. L'Union Européenne a imposé que le transport international de voyageurs ne soit plus considéré comme mission de service public. De ce fait, il ne peut théoriquement plus être financé par les pouvoirs publics. En Belgique, l'État Fédéral a adopté la même position pour le transport national à grande vitesse (article 156 de la loi du 21/3/1991 sur la réforme de certaines entreprises publiques économiques et articles 3 et $10 \mathrm{du}$ deuxième contrat de gestion entre la SNCB et l'État).

19. De source SNCB, le prix final d'une rame Thalys PBKA est de 27,27 millions d'euros $(1,1$ milliards BEF) + $15 \%$ pour le préfinancement.

20. Avant le remplacement des vols Air France par des Thalys et la faillite de la Sabena.

\section{RÉSUMÉS}

La mode est à l'interconnexion des réseaux TGV et des aéroports. Si dans certains cas cela est pleinement justifié par une aire d'influence de l'aéroport suffisamment étendue pour correspondre aux distances TGV, dans d'autres ce n'est pas le cas et la correspondance avion / TGV est alors peu utilisée par les voyageurs.

Qu'en est-il du cas de l'aéroport de Bruxelles-National ? Cet article se propose d'analyser l'utilité d'une desserte TGV de cet aéroport, du point de vue de l'offre et de la demande en transport, mais également en tenant compte des enjeux politiques et environnementaux et des contraintes 
techniques et économiques à prendre en compte.

Il apparaît alors qu'une telle desserte n'est sans doute pas nécessaire à court terme, et qu'à plus long terme elle dépend certes de l'évolution de l'offre et de la demande, mais également de choix de société, donc d'arbitrages politiques.

The present trend favours the interconnection between high-speed trains and airports. If this is fully justified in some cases when the airport has a sufficiently wide sphere of influence to cover the high-speed trains distances. In other cases it is not, so that the air-HST connection is not much used by the passengers.

What about the Brussels-National airport? This article proposes to analyse the usefulness of a TGV link to the airport, considering supply and demand in the field of transport, but also the political and environmental stakes as well as the technical and economic constraints to be taken into account.

It then appears that such a link is probably not necessary in the short time and that in a longer time it will depend of course on how supply and demand evolve but also on society choices, in other words political arbitration.

\section{INDEX}

Mots-clés : géographie des transports, transports internationaux, intermodalité, multimodalité, interconnexion, TGV, aéroport, Bruxelles

Keywords : transport geography, international transport, intermodality, multimodal transport, interconnection, HST, airport, Brussels

\section{AUTEUR}

\section{FRÉDÉRIC DOBRUSZKES}

Institut de Gestion de l'Environnement et d'Aménagement du Territoire, Université Libre de Bruxelles, Frederic.Dobruszkes@ulb.ac.be 